\title{
MedienPädagogik
}

\section{Editorial: Neue Fernsehserien und ihr Potenzial für eine kritische Medienpädagogik}

\author{
Elena Pilipets und Rainer Winter
}

Die Auseinandersetzung mit Fernsehserien spielt eine wichtige Rolle im Leben vieler Menschen weltweit: Die Serien wiederholen und entwickeln sich, sind Teil der Medienroutine, begleiten uns im Alltag. Was sie erzählen, entsteht daher nicht unerwartet oder zufällig, sondern verweist auf eine langfristige Ausprägung populärkultureller Ausdrucksformen, die kognitiv und ästhetisch sowie anwendungsorientiert ist (Eichner et al. 2013). Nichtsdestotrotz hatten die populären Serien schon immer unvorhersehbare Folgen». Vor allem Fernsehserien, die angesichts enormer Veränderungen in verschiedenen Bereichen der kulturindustriellen Medienunterhaltung seit den 1980er- und vor allem den 1990er- Jahren als anspruchsvolle und experimentelle erzählerische Formen wiederentdeckt wurden, scheinen sich permanent aufs Neue zu erfinden. Episode für Episode, Staffel für Staffel, irgendwo im Dazwischen von «coming next...» und «previously on» (Meteling et al. 2010) entfalten sich komplexe mediale Erfahrungswelten, indem sie dem performativen Imperativ popkultureller Überbietung zufolge an der kontinuierlichen Erweiterung ihrer narrativen und medialen Möglichkeiten arbeiten (Jahn-Sudmann/Kelleter 2012). Diese prozessorientierte Fähigkeit der Fernsehserie, aus der eigenen Transformation zu lernen, wird im Rahmen dieses Themenheftes hinsichtlich folgender (medien-)pädagogischer Aspekte diskutiert:

\section{Serialität als Geburtsstätte der popkulturellen (Medien-)Kompetenz}

Das serielle Prinzip der variierenden Wiederholung fungiert als konstitutives Merkmal gegenwärtiger medialer Transformationsprozesse und kommerzieller Reproduktionsprozesse (Beil/Engell/Schröter/Schwaab/Wentz 2012). Gleichzeitig entwickeln sich auch kulturelle Praktiken und Lernprozesse performativ ausgehend von den Abweichungen in der alltäglichen Wiederholung und Wiederaneignung (Düllo 2011). Populäre Medien- und Unterhaltungsangebote unterhalten dementsprechend nicht nur, sie erziehen auch, wie Henry Giroux (2002) feststellt. Die aktuelle Vielfalt an Verwendungskontexten, Sinnangeboten und medialen Vernetzungen serieller Medienerzählungen veranschaulicht einen komplexen und prozessorientierten Kompetenzerwerb im Umgang mit dem Phänomen Fernsehserie. Marginale Erscheinungen und subkulturelle Werte werden dabei im selben Ausmaß artikuliert wie dominante Ideologien und so zum Gegenstand alläglicher Aushandlungspraktiken, die sich auf verschiedenen Ebenen abspielen (Winter 2013). 
Ausgehend von dieser konstitutiven Ambivalenz demonstriert Gerald Poscheschnik in seinem Beitrag anhand einer psychoanalytischen Untersuchung von Game of Thrones (HBO, USA, 2011-), inwiefern Fernsehserien als Artikulationsformen gesellschaftspolitischer Phantasien fungieren können. Da Serien sowohl bewusste als auch unbewusste Elemente in sich bergen, enthalten sie keine eindeutig identifizierbare Moral, die versuchen würde, das Publikum auf plumpe Art und Weise zu erziehen. Game of Thrones lässt aus diesem Grund keine vereinfacht moralisierenden Interpretationen zu. Indem die Serie ihr Publikum nicht nur unterhält, sondern auch verstört, entzieht sie sich einer simplifizierenden, automatisch ablaufenden Deutung. Stattdessen werden die Zuschauer/innen sehr wohl stark herausgefordert, Bedeutungen durch individuelle Denk- und kollektive Kommunikationsprozesse zu konstruieren, wodurch bewusste aber auch affektive Reflexionsräume eröffnet werden.

Die damit einhergehende Kompetenzbildung ist von den produkt-, lebensstil- und gebrauchsspezifischen Vorlieben des seriellen Charakters nicht zu trennen und gehört somit zur selbstverständlichen und habitualisierten Mediensozialisation. Die zeitgenössischen Serienformate funktionieren nach der populären Bricolage-Logik und profitieren von der kontinuierlichen Differenzerzeugung. Ihre provokanten Themen, komplexen Erzählwelten, vielschichtigen Figurenensembles und intertextuellen Querverweise laden zur wiederholten Rezeption ein, irritieren, motivieren und begeistern. Zum anderen verlangen und fördern sie genau jene Fähigkeiten, die den Alltag im Zeitalter allumfassender medialer Vernetzung, geprägt durch ein Zuviel an Information, Stress, Gefahr von Prekarität und Verlust an Sicherheit, kennzeichnen. So beschäftigt sich Marcus S. Kleiner anhand der Analyse US-amerikanischer Fernsehserien 24 (Fox, USA, 2001-2010) und Sleeper Cell (Showtime, USA, 2005-2006) mit den medialen und performativen Eigenschaften des Phänomens der seriellen «Terrorbildung». Als die ersten Serien, die die permanente TerrorBedrohung und Terror-Bekämpfung explizit zum Leitthema machen, werden 24 und Sleeper Cell auf ihre Fähigkeit hin überprüft, soziale Bilder sowie Diskurse über den Terrorismus auf eine spezifische Art zu artikulieren. Diese trägt einerseits zur sozialen Gewöhnung an den Terror und seine medialen Bilder bei, bietet zur selben Zeit aber auch Ressourcen zur kritischen Auseinandersetzung mit der medialen Mitgestaltung des Terrorismus im Kontext gesellschaftlicher Entwicklungen nach 9/11.

Die performative Struktur serieller Medienerzählungen sowie ihre Ambivalenz und Alltagsrelevanz thematisiert im Hinblick auf Transformations- und Aushandlungscharakter kultureller Bildungsprozesse die Analyse von Olaf Sanders. Ausgehend von einem durch amerikanische Fernsehserien wie True Detektive (HBO, USA, 
2014-), Justified (FX, USA, 2010-2015) oder Sons of Anarchy (FX, USA, 2008-2014) mobilisierten Spannungsverhältnis zwischen Repräsentationen des globalen Hillbillytums und seinen lokalen ethnozentrischen Abwandlungen, hebt Sanders die historische Ambivalenz der ländlichen, mit Strukturen der Dominanz artikulierten, ironisch gebrochenen Hillbilly-Figur hervor. An theoretische Überlegungen von Adorno, Ranciere und Deleuze anknüpfend, wird dabei eine durch differenzierte serielle Abhandlungen geformte «Schule der Ambivalenz» nachgezeichnet, die über wiederholte Mobilisierungen des popkulturellen Bedeutungsreichtums ein Rhizom aus links- und rechtskodierten alltagspolitischen Auseinandersetzungen bildet.

\section{Gelebte Komplexität der Serie im digitalen Medienalltag}

Durch Prozesse der Digitalisierung hat sich die von Klaus Theweleit bereits in den $90 e r$ Jahren beobachtete «Verschiebung von Massenphänomenen auf Serienphänomene» $(1998,223)$ stark intensiviert. Längst sind die televisuellen Narrative nicht mehr auf die linearen Eigenschaften des herkömmlichen Fernsehens angewiesen, sondern verbreiten sich auf verschiedenen Wegen in einer heterogenen Medienlandschaft konvergenter Kommunikationsplattformen, mobiler Endgeräte und sozialer Netzwerke (Klein 2013). Die serienbezogene Partizipationskultur generiert polyseme Medientexte, die in unterschiedlichen Kontexten für unterschiedliche Menschen auf unterschiedliche Weise funktionieren müssen, stets angeeignet werden wollen und dementsprechend vielfältig praktiziert, genutzt und gelebt werden. Indem sie sich immer mehr an der Konstruktion alltäglicher Medienwirklichkeiten beteiligen, weisen die narrativen Welten zeitgenössischer Serien einen höheren Grad an Komplexität auf. Diese gelebte Komplexität der Serie entfaltet sich durch das Sammeln, Tauschen, Verschieben und Kombinieren von populär ausgehandelten und medial vermittelten Wissensformen. Wie populäre Serien in ihrer praxisorientierten Anwendungsvielfalt sowohl die Selbst-Expression als auch die Gemeinschaftsbildung performativ herstellen, thematisiert am Beispiel von Fan-Fictions der Beitrag von Julia Elena Goldmann. Dabei wird ausgehend von fanbasierten Praktiken der Auseinandersetzung mit Supernatural (The WB, USA, 2005-) verdeutlicht, wie transformative Prozesse der Alltagsbewältigung durch Aneignungen des fiktionalen Formats von dieser und anderen Vampirserien erweitert und kreativ verhandelt werden.

Die gelebte Komplexität zeitgenössischer serieller Formate verbindet das interaktive Potenzial global vernetzter, digitaler Umgebungen mit den narrativen Möglichkeiten des Fernsehens, wobei die Eigenart dieser Beziehungen gleichzeitig als Produkt und Praxis der seriellen Erzählung selbst reflektiert wird. Davon zeugt 
die gegenwärtige Herausbildung einer transnationalen Serienkultur (Eichner et al. 2013), die sich durch enorme Vielfalt, Konnektivität, Geschwindigkeit und Hybridität auszeichnet. Gleichzeitig verlangt sie aber sowohl seitens der Produzent/innen als auch Rezipient/innen nach Nutzungs- und Aneignungskompetenzen, die dem neoliberalen Produktivitätszwang unter Bedingungen einer allumfassenden Konnektivität entspringen. Multitasking, situative Rückkoppelung und verteilte Informationsabwicklung sind mittlerweile wichtige Voraussetzungen der Serienrezeption (Kelleter 2012), betreffen jedoch genauso die Bedingungen des medienseriellen Erzählens selbst. Wie sie durch die narrativen Möglichkeiten von aktuellen, auf einen globalen Markt ausgelegten Serienproduktionen problematisiert werden, zeigt die von jan jagodzinski aus Gilles Deleuzes symptomatologischer Position durchgeführte Analyse der ersten Staffel von Mr. Robot (USA Network/Amazon, USA, 2015-). Am Beispiel der Hacker-Hauptfigur Elliot Anderson wird dargelegt, inwiefern die Serie Einblicke in aktuelle Dynamiken der Medienkontrollgesellschaft gewähren kann. Medienpädagogische Aspekte, die aus der Konfrontation des Publikums mit ethischen Problemen der digitalen Überwachung entstehen, werden dabei im Hinblick auf Reflexions- und Transformationspotenziale der Serie hervorgehoben.

Alternative Formen der Vernetzung zwischen den Aneignungspraktiken der Serienproduzent/innen, narrativen Strategien konvergenter Serienformate, und der Rolle transnational aktiver Fangemeinschaften bei der Entwicklung medialer und kommunikativer Kompetenzen diskutiert der Beitrag von Elena Pilipets, Matthias Wieser und Rainer Winter. Als Ausgangspunkt dafür dient die gelebte Komplexität der interaktiven, deutsch-französischen Miniserie About:Kate (Arte, D/F, 2013), die aus interdisziplinärer Perspektive im Zusammenhang mit der Entstehung einer sich zunehmend transmedial entfaltenden Serienkultur diskutiert wird. Im Fokus steht die Serie als Schnittstelle des intermediären und soziokulturellen Austauschs, die, um funktionieren zu können, über text-, kontext- und plattformübergreifende Erweiterungen in alltägliche Praktiken der Mediennutzung eingebunden werden muss.

\section{Die Kompetenz der Serie und ihr Potenzial für kritische Medienpädagogik}

Die kritische Medienpädagogik, die sich vor allem in den USA entwickelt hat (Winter 2006a), begreift populäre Medienprodukte als kulturelle Praktiken und Ereignisse, in denen aktuelle politische Auseinandersetzungen, gesellschaftliche Trends und soziale Konflikte zum Ausdruck gebracht werden. Betrachtet man Bildungsprozesse aus dieser Perspektive, so geht es in erster Linie um ihre Transformationspotenziale, die erst eingebunden in Praktiken, Routinen und Vergnügen im 
Umgang mit Medien-, Populär- und Konsumkultur angemessen verstanden werden können (Giroux 2001). Die Einsicht, dass Fernsehserien als Produkte soziokultureller und medientechnischer Transformationen fungieren, zum anderen aber auch eine aktive Rolle in diesen Prozessen spielen (Winter 2006b), veranschaulicht der Beitrag von Dennis Klinkhammer. Mittels einer medialen Längsschnittanalyse von Star Trek wird mit dem Fokus auf die Frage der Repräsentation von Menschen mit Behinderung ein Paradigmenwechsel von exklusiver Fremdbestimmung in den 60 ern über idealtypische Inklusionsverhältnisse in den 70ern und 80ern zu einer tendenziell selbstbestimmten Darstellung von Behinderung in den 90ern nachgezeichnet. Dabei wird aufgezeigt, dass solche seriell auf Dauer gestellten Repräsentationen nicht nur einen gesellschaftlichen Zustand, sondern vor allem einen gesellschaftlichen Prozess darstellen, dessen retrospektive Betrachtung ein besseres Verständnis von gesellschaftlichen Inklusions- und Exklusionsstrukturen und ihren Voraussetzungen ermöglichen kann.

Populäre Serien, die aufgrund ihrer performativen Struktur in einem ständigen Wechselverhältnis von Selbstreferenz, Selbsterzählung und Selbstmotivation gleichzeitig auf eigene bestehende Muster und zukunftsorientierte Entwicklungsmöglichkeiten verweisen, sind somit nicht nur als ausgezeichnete pädagogische Hilfsmittel zu begreifen, sondern weisen ihre eigene Lernkompetenz auf. Indem sie sich selbst stets neu definieren, tragen sie auf mehreren, oft indirekten Wegen, dazu bei, die aktuelle gesellschaftliche Situation zu hinterfragen, herauszufordern und nach Alternativen zu suchen. Die unkonventionellen Erzählungen zeitgenössischer Fernsehserien übersetzen das Einmalige in das Allägliche, das Routinierte in das Einzigartige. Ihre strukturelle Wiedererkennbarkeit geht oft mit Irritation und Bedeutungsverschiebung einher, wodurch repräsentative Bedeutungsstrukturen, wie Christian Toth am Beispiel der südkoreanischen Reality-TV-Serie The Return of Superman (KBS, Kor, 2013-) veranschaulicht, ihrer Selbstverständlichkeit beraubt und in alltäglichen Praktiken transformiert werden können. Ausgehend von der Fähigkeit der Serie, in ihrer Abhandlung traditioneller paternalistischer Erziehungsstrukturen Südkoreas alternative Repräsentationen eines angestrebten Rollen- und Familienverständnisses von Vätern zur Verfügung zu stellen, wird demonstriert, inwiefern diese gleichzeitig hinsichtlich ihrer gesellschaftspolitischen Steuerungsfunktion mobilisiert werden. In serienpädagogischen Prozessen kann somit aufgezeigt werden, wie solche Medienerzählungen in der doppelten Funktion einer Unterhaltungs- und Dokumentationsnarration als sich wiederholende Praktiken des Kultur- und Medienkonsums das alltägliche Leben mitgestalten. 
Indem sie auch skurrile und abweichende Subjektpositionen und Lebensstile zur Aufführung bringen, stellen die Serien zunehmend mehrdeutige Assoziations- und Identifikationsangebote zur Verfügung. Vertraute Zeichen, Gegenstände, Praktiken und Bilder der populären Alltagskultur werden dabei in oft überraschenden und respektlosen Zusammenhängen erfahrbar gemacht, wobei anti-elitäre und tendenziell ungewöhnliche Wissensformen und Lernsituationen ermöglicht werden. Der Frage, inwiefern diese außergewöhnlichen Thematiken an das Alltagsleben ihres Publikums anschließen, zeigt Daniela Bruns in ihrer Analyse von Dexter (Showtime, USA, 2006-2013). Indem sie der Frage nachgeht, wie neoliberale Subjektpositionen mit Hilfe von kontroversen Charakteren vermittelt, aber auch umgedeutet und kritisiert werden, stellt sie die Möglichkeiten serieller Erzählungen heraus, eine bedeutsame Auseinandersetzung mit einem reflektierten und an einem Austausch interessierten Publikum hervorzubringen. Da Dexter mit einem sympathischen Serienkiller als Hauptfigur nicht vorgibt, was richtig und was falsch ist, sondern die Zuschauer/innen in emotionale und ethische Widersprüche verwickelt, artikuliert die Serie Kritik durch die Hervorbringung von Irritation, während das Publikum über Reflexion der eigenen Haltung Anknüpfungspunkte für alltagsrelevante Veränderungsprozesse schafft.

Eine kritische Analyse von Fernsehserien ermöglicht somit den Zugang zu konfliktreichen kulturellen, sozialen und historischen Kontexten, die in die seriellen Produkte und Praktiken gegenwärtiger populärer Medienkultur bereits eingeschrieben sind, durch diese jedoch stets auch neu hervorgebracht und ausgehandelt werden. Die Attraktivität der Fernsehserien für eine kritische Medienpädagogik liegt dabei auf der Hand, wirft aber auch zahlreiche Fragen auf, die im Rahmen dieses Themenheftes aus verschiedenen Perspektiven anhand mehrerer Beispiele diskutiert werden: Inwiefern erlaubt die Wiederholung als Lernprinzip das Artikulieren von Kritik mittels Populärkultur? Welche Rolle spielt dabei das Vergnügen im Umgang mit seriellen Angeboten? Wie hängen zeitgenössische Fernsehserien mit größeren historischen Kontexten und gesellschaftlichen Transformationen zusammen? Welche Ressourcen stellen die Serienkulturen in der Nutzung neuer Medienangebote wie auch in Prozessen der Bedeutungskonstruktion, kultureller Orientierung und Sinnbildung dar? Vor diesem Hintergrund beleuchten die Beiträge nicht nur die narrativen Besonderheiten von, sondern auch Aspekte des alltäglichen Umgangs mit den Serien. Damit zeigen sie das prozess- und beziehungsorientierte Potenzial der damit einhergehenden populären Kompetenzen auf, sowie die Möglichkeiten der Umsetzung seiner affektiven und performativen (Lern-)Eigenschaften in medienpädagogischen Kontexten. 


\section{Literaturhinweise}

Beil, Benjamin, Lorenz Engell, Jens Schröter, Herbert Schwaab, und Daniela Wentz. 2012. «Die Fernsehserie als Reflexion und Projektion des medialen Wandels.» In Mediatisierte Welten: Beschreibungsansätze und Forschungsfelder, hrsg. v. Andreas Hepp und Friedrich Krotz, 197-223, Wiesbaden: VS.

Düllo, Thomas. 2011. Kultur als Transformation. Eine Kulturwissenschaft des Performativen und des Crossover. Bielefeld: transcript.

Eichner, Susanne, Lothar Mikos, und Rainer Winter. Hrsg. 2013. Transnationale Serienkultur. Theorie, Ästhetik, Narration und Rezeption neuer Fernsehserien. Wiesbaden: Springer VS.

Giroux, Henry Armand. 2001. Theory and Resistance in Education. Towards a Pedagogy for the Opposition. Westport, London: Bergin \& Garvey.

Giroux, Henry Armand. 2002. Breaking Into the Movies. Film and the Culture of Politics. Massachusetts, Oxford: Blackwell.

Jahn-Sudmann, Andreas, und Frank Kelleter. 2012. «Die Dynamik serieller Überbietung: Zeitgenössische amerikanische Fernsehserien und das Konzept des Quality TV.» In Populäre Serialität: Narration-Evolution-Distinktion. Zum seriellen Erzählen seit dem 19. Jahrhundert, hrsg. v. Frank Kelleter, 205-224, Bielefeld: Transcript.

Kelleter, Frank. 2012. «Serien als Stresstest» Frankfurter Allgemeine Zeitung, 30: 31.

Klein, Thomas. 2013. «Von der Episode zur Webisode. Serialität und mediale Differenz.» In Medien. Erzählen. Gesellschaft: Transmediales Erzählen im Zeitaler der Medienkonvergenz, hrsg. v. Karl Nikolaus Renner, Dagmar Hoff und Matthias Krings, 118-138. Berlin: Walter de Gruyter.

Meteling, Arno, Isabell Otto, und Gabriele Schabacher. Hrsg. 2010. Previously On... Zur Ästhetik der Zeitlichkeit neuerer TV-Serien. München: Wilhelm Fink.

Theweleit, Klaus. 1998. Ghosts. Drei leicht inkorrekte Vorträge. Frankfurt am Main: Basel.

Winter, Rainer. 2006a. «Kultur, Reflexivität und das Projekt einer kritischen Pädagogik.»In Cultural Studies und Pädagogik. Kritische Interventionen, hrsg. v. Paul Mecheril und Monika Witsch, 21-50, Bielefeld: Transcript.

Winter, Rainer. 2006b. «Television Studies im 21. Jahrhundert. Für eine kritische Analyse der Fernsehkultur.» In Informationen zu Deutschdidaktik 2: 18-25.

Winter, Rainer. 2013. «Fernsehserien als Kult. Vom klassischen Medienkult zu den Strategien der globalen Kulturindustrie.» In Transnationale Serienkultur. Theorie, Ästhetik, Narration und Rezeption neuer Fernsehserien, hrsg. v. Susanne Eichner, Lothar Mikos und Rainer Winter, 67-87, Wiesbaden: Springer VS. 